\title{
Formulating the Net Gain of MISO-SFN in the Presence of Self-Interferences
}

\author{
Sungho JEON ${ }^{12}$, Junghyun KIM ${ }^{1}$, Ha-Kyun MOK ${ }^{1}$, Jong-Soo SEO ${ }^{2}$ \\ ${ }^{1}$ Technical Research Institute, Korean Broadcasting System (KBS), Seoul, 150-790, Republic of Korea \\ ${ }^{2}$ Department of Electrical and Electronic Engineering, Yonsei University, Seoul, 120-749, Republic of Korea \\ jsseo@yonsei.ac.kr
}

\begin{abstract}
In this study, an analytical formula for multipleinput single-output single frequency network gain (MISOSFNG) is investigated. To formulate the net MISO-SFNG, we derived the average signal to interference plus noise ratio (SINR) where the gain achieved by the distributed MISO diversity as a function of power imbalance is curve-fitted. Further, we analyzed the losses owing to self-interferences resulting from the delay spread and imperfect channel estimation. We verified the accuracy and effectiveness of the derived formula by comparing the measurement results with the analytical results. The derived formula helps to understand how various system factors affect the gain under a given condition. The formula can be used to evaluate the $M I S O-S F N G$ and to predict the MISO-SFN coverage in various system configurations.
\end{abstract}

\section{Keywords}

Single frequency network (SFN), DVB-T2 MISO processing, power imbalance, calibrated coverage prediction

\section{Introduction}

In a multiple frequency network (MFN), each transmitter occupies a specific frequency, and the service coverage is determined by the specific transmitter. Coverage prediction in MFNs is mainly carried out by calculating the path loss between the transmitter and the receiving point, and the coverage contour is obtained by lining the points where the receive field strength (in $\mathrm{dBm}$ or $\mathrm{dB} \mu / \mathrm{m}$ ) is equal to the minimum required field strength. Consequently, applying an appropriate path loss model is important for an accurate prediction of service coverage.

In a single frequency network (SFN), multiple transmitters share one frequency, and therefore, the service coverage is determined based on those transmitters [1]. The field strength and service coverage in the overlapping area of the received signal in the SFN increase owing to the SFN gain (SFNG) as shown in Fig. 1. Therefore, accurately estimating the SFNG is necessary to predict and determine the SFN service coverage. However, according to [4], a theoretical closed-form formula relevant to SFNG calculation has yet to be developed, although most results on SFNG have been collected and collated by laboratory experiments and field measurement campaigns [5].

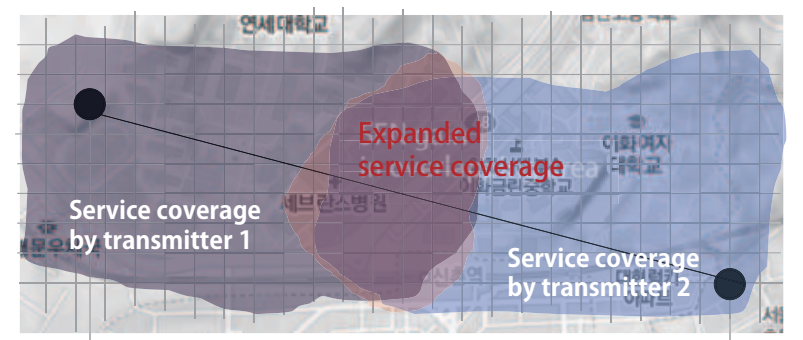

(a) Service coverage of SFN

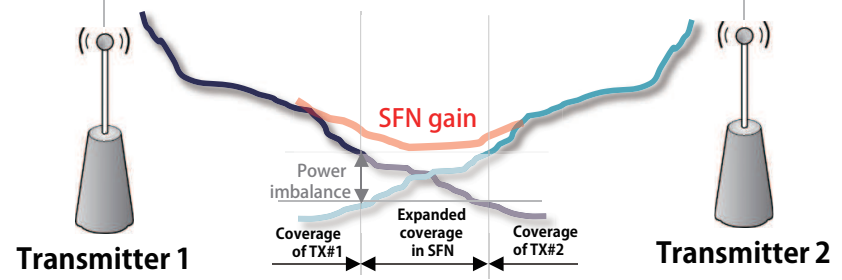

(b) Field strength of SFN

Fig. 1. Two equal power SFNs improve the service coverage by means of SFN gain.

Currently, two types of SFN are available: 1) the single-input single-output SFN (SISO-SFN), which is typically used in the first-generation broadcasting systems [6] such as DVB-T; 2) the multiple-input single-output SFN (MISO-SFN) based on the modified Alamouti code [21][14], which is newly introduced in the second-generation broadcasting system [7] such DVB-T2 [8]. In the SISO-SFNG case, the simple aggregate power sum calculation has been verified through a wide range of field measurements in many papers [13][10]. Recently, an analytical formula that considers SISO-SFN loss (SISO-SFNL) due to the erasure effect [11] has been developed [12].

Compared to the conventional SISO-SFN, MISO-SFN provides a higher SFN gain owing to the transmit diversity in overlapping service coverage areas [13]-[17]. The SISOSFNG can be obtained without any signal processing at the receiver, and therefore, the gain can be predicted simply by adding the power of the received signals from all the trans- 


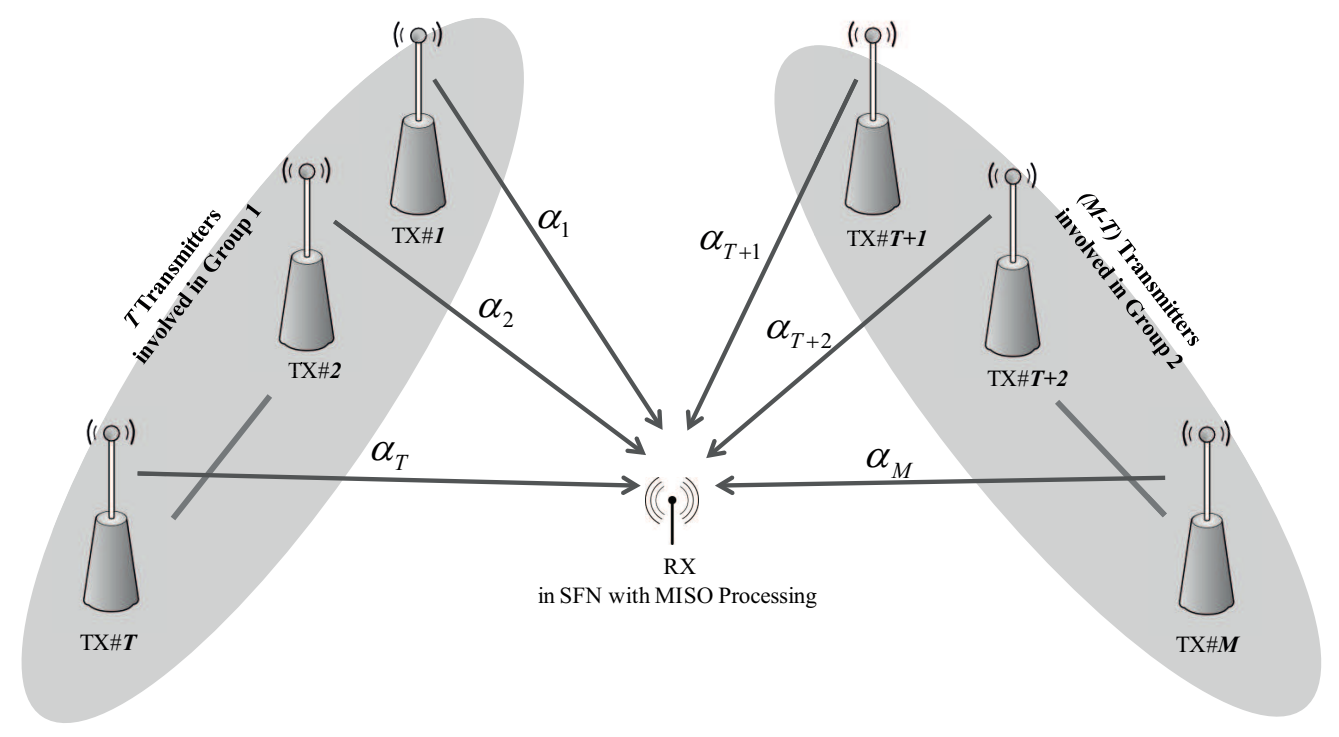

Fig. 2. A general MISO-SFN configuration [13][4].

mitters. On the other hand, the MISO-SFN gain (MISOSFNG) requires mandatory signal processing at the receiver specified in the standard as the gain cannot be predicted directly.

Thus, to the best of our knowledge, the potential and practical MISO-SFNG has been predicted only through simulations [14], laboratory experiments [15], and field measurements [16][17]. Although the measurement data reported in these studies satisfactorily confirm the phenomenon, there exists no commonly accepted method or formula for evaluating MISO-SFNG explaining the various system factors influencing the gain under a given condition. This is because it is inherently impossible to control the test environment completely during the measurements, even by following the standardized measurement process [18][19]. Therefore, measurement results obtained by using different techniques such as the channel estimation and interpolation method, and the synchronization strategy, would differ from each other.

Nevertheless, the analysis of the measured data reported in previous studies show that the practical MISOSFNG is dependent on the transmission parameters, including the modulation order and code rate, power imbalance and relative delay between the transmitters, and pilot pattern related channel estimation errors. Therefore, an appropriate analytical tool is required to verify the measurement results and explain such phenomena.

In this paper, an analytical formula for MISO-SFNG is presented. In order to formulate the net MISO-SFNG, we first derive the analytical formula for the average signal to interference plus noise ratio (SINR) by considering the gain of the distributed MISO diversity as well as the losses due to the self-interferences resulting from the delay spread and imperfect channel estimation. The accuracy and effectiveness of the derived formula is verified by comparing the measured results with the numerical results.

\section{Signal Model in MISO-SFN}

\subsection{Transmitted Signal Model According to MISO Processing}

To enhance the SFNG in the coverage overlapping area, DVB-T2 adopts the MISO Alamouti code [21] in the frequency domain, i.e., a distributed space-frequency block code (SFBC), while the receiver can still use a single antenna [8]. Let $X_{n, k}^{a}$ be the symbol on two consecutive subcarriers $k$ and $k+1$ of the orthogonal frequency division multiplexing (OFDM) symbol $n$ transmitted on group $a=1,2$. In the symbol stream $X_{n, k}$, the transmitted symbols are organized as follows:

$$
\begin{aligned}
& X_{n, 2 k}^{1}=X_{n, 2 k}, \quad X_{n, 2 k+1}^{1}=X_{n, 2 k+1}, \\
& X_{n, 2 k}^{2}=-X_{n, 2 k+1}^{*}, \quad X_{n, 2 k+1}^{2}=X_{n, 2 k}^{*}
\end{aligned}
$$

where the transmitter in group 2 swaps the odd and even cells of the OFDM symbol and introduces the complex conjugate $(\cdot)^{*}$ and a sign change. The $K$-point inverse discrete Fourier transform (IDFT) of $X_{n, k}^{a}=\frac{1}{\sqrt{K}} \sum_{k=0}^{K-1} x_{n, k}^{a} e^{j 2 \pi k \Delta f}$ yields the discrete time-domain OFDM symbol where the number of subcarriers $K$ and the subcarrier spacing $\Delta f$ are specified in the standards, i.e., Table 66 in DVB-T2 [8].

\subsection{Received Signal Model in Matrix Form}

As illustrated in Fig. 2, the index sets for $M$ transmitters in MISO-SFN are represented by $\mathcal{G}_{1}=\{1,2, . ., T\}$ for group 1 and $G_{2}=\{T+1, T+2, . ., M\}$ for group 2 . The transmitted signal $x_{n}^{a}[t]$ is filtered by the discrete-time channel impulse response $h_{i}[t]$ and contaminated by the additive noise $w_{n}[t]$; therefore, the received signal $y_{n}[t]$ in MISO-SFN can be defined as [17] 


$$
\begin{aligned}
y_{n}[t]= & \sum_{i \in \mathcal{G}_{1}} \alpha_{i} h_{i}\left[t-\Delta \tau_{i}\right] \circledast x_{n}^{1}[t] \\
& +\sum_{i \in \mathcal{G}_{2}} \alpha_{i} h_{i}\left[t-\Delta \tau_{i}\right] \circledast x_{n}^{2}[t]+w_{n}[t]
\end{aligned}
$$

where $\alpha_{i}$ and $\Delta \tau_{i}$ represent the amplitude and the relative delay of the received signal from each transmitter in the SFN, and $\circledast$ denotes the convolution operator. $x_{n}^{1}[t]$ represents the transmitted signal from group 1 and $x_{n}^{2}[t]$ denotes the corresponding MISO encoding in (2) to the original signal transmitted by group 2 .

$H_{n, k}^{a}$ denotes the channel frequency response after OFDM demodulation for group $a$. The two received signals $Y_{n, 2 k}$ and $Y_{n, 2 k+1}$ from two transmitter groups using a single antenna in two consecutive subcarriers $k$ and $k+1$ are represented as [8][20]

$$
\begin{aligned}
Y_{n, 2 k} & =H_{n, 2 k}^{1} X_{n, 2 k}-\alpha H_{n, 2 k}^{2} X_{n, 2 k+1}^{*}+W_{n, 2 k} \\
Y_{n, 2 k+1} & =H_{n, 2 k+1}^{1} X_{n, 2 k+1}+\alpha H_{n, 2 k+1}^{2} X_{n, 2 k}^{*}+W_{n, 2 k+1}
\end{aligned}
$$

where $\alpha$ is the power imbalance between transmitter group 1 and group 2, represented by

$$
\alpha=\sqrt{\left|\sum_{i \in \mathcal{G}_{1}} \alpha_{i}^{2}-\sum_{i \in \mathcal{G}_{2}} \alpha_{i}^{2}\right|} .
$$

$W_{n, 2 k}$ and $W_{n, 2 k+1}$ represent the additive noise with a complex circularly symmetric Gaussian random variable with zero mean and variance $\sigma_{w}^{2}$. We can represent (4) in the following matrix form:

$$
\underbrace{\left[\begin{array}{c}
Y_{n, 2 k} \\
Y_{n, 2 k+1}^{*}
\end{array}\right]}_{\mathbf{Y}}=\underbrace{\left[\begin{array}{cc}
H_{n, 2 k}^{1} & -\alpha H_{n, 2 k}^{2} \\
\alpha H_{n, 2 k+1}^{2 *} & H_{n, 2 k+1}^{1 *}
\end{array}\right]}_{\mathbf{H}} \underbrace{\left[\begin{array}{c}
X_{n, 2 k} \\
X_{n, 2 k+1}^{*}
\end{array}\right]}_{\mathbf{X}}+\underbrace{\left[\begin{array}{c}
W_{n, 2 k} \\
W_{n, 2 k+1}^{*}
\end{array}\right]}_{\mathbf{W}} .
$$

In the distributed MISO structure of the MISO-SFN system, $H_{n, 2 k}^{a}$ and $H_{n, 2 k+1}^{a}, a \in\{1,2\}$ may be independent because of the fully separated transmit antennas. Note that the transmit correlation of two antennas is obtained by

$$
\eta=E\left[H_{n, 2 k}^{1} H_{n, 2 k}^{2 *}\right]=E\left[H_{n, 2 k+1}^{1} H_{n, 2 k+1}^{2 *}\right] \approx 0
$$

where $E[\cdot]$ is the expectation operator.

\subsection{Detected Signal Model with Channel Esti- mation Error}

In order to estimate the channel response of each OFDM symbol in the frequency domain, the receiver measures the channel using pilot symbols and then interpolates between these measurements [20]. As a result, the estimated channel matrix $\hat{\mathbf{H}}$ is obtained by [23]

$$
\hat{\mathbf{H}}=\mathbf{H}+\underbrace{\left[\begin{array}{cc}
e_{n, 2 k}^{1} & -e_{n, 2 k}^{2} \\
e_{n, 2 k+1}^{2 *} & e_{n, 2 k+1}^{1 *}
\end{array}\right]}_{\mathbf{E}}
$$

where $\mathbf{E}$ is a random matrix with each element $e_{n, k}^{a}$ being an i.i.d complex Gaussian random variable $\mathcal{C} \mathcal{N}\left(0, \sigma_{e}^{2}\right)$ and the amount of error is dependent on the pilot patterns as well as the estimation and interpolation methods [20].

Using the estimated channel coefficients, a matched filtering $\hat{\mathbf{H}}^{H}$ for maximum likelihood decoding is applied [21], and the detected signal is given by

$$
\begin{aligned}
\mathbf{Z}= & \hat{\mathbf{H}}^{H} \mathbf{Y}=\left(\mathbf{H}^{H}+\mathbf{E}^{H}\right)(\mathbf{H X}+\mathbf{W}) \\
= & {\left[\begin{array}{c}
\left(\left|H_{n, 2 k}^{1}\right|^{2}+\alpha^{2}\left|H_{n, 2 k+1}^{2}\right|^{2}\right) X_{n, 2 k} \\
\left(\left|H_{n, 2 k+1}^{1}\right|^{2}+\alpha^{2}\left|H_{n, 2 k}^{2}\right|^{2}\right) X_{n, 2 k+1}^{*}
\end{array}\right] } \\
& +\alpha\left[\begin{array}{c}
\left(H_{n, 2 k+1}^{1 *} H_{n, 2 k+1}^{2}-H_{n, 2 k}^{1 *} H_{n, 2 k}^{2}\right) X_{n, 2 k+1}^{*} \\
\left(H_{n, 2 k+1}^{1} H_{n, 2 k+1}^{2 *}-H_{n, 2 k}^{1} h_{n, 2 k}^{2 *}\right) X_{n, 2 k}
\end{array}\right] \\
& +\mathbf{E}^{H} \mathbf{H X} \\
& +\left(\mathbf{H}^{H}+\mathbf{E}^{H}\right) \mathbf{W}
\end{aligned}
$$

where (9) is the desired signal components, (10) leads to self-interference due to delay effects, (11) is caused by channel estimation error, and (12) are the effective additive noises.

\section{Formulating the Net MISO-SFNG}

\subsection{Gain in Flat Fading Channel with Perfect Channel Estimation}

For evaluating a pure MISO-SFNG, perfect channel estimation is assumed, i.e., $\mathbf{E}=\mathbf{0}_{2}$, where $\mathbf{0}_{r}$ is an $r \times r$ zero matrix. In addition, two consecutive subcarriers are assumed to suffer from flat Rayleigh fading where $H_{n, 2 k}^{1}=H_{n, 2 k+1}^{1}$ and $H_{n, 2 k}^{2}=H_{n, 2 k+1}^{2}$. Therefore, the resulting channel matrix $\mathbf{H}$ is orthogonal as explained by the following equation:

$$
\mathbf{H}^{H} \mathbf{H}=\left(\left|H_{n, 2 k}^{1}\right|^{2}+\alpha^{2}\left|H_{n, 2 k}^{2}\right|^{2}\right) \mathbf{I}_{2}
$$

where $\mathbf{I}_{r}$ represents an $r \times r$ identity matrix.

In contrast to the uncoded performance [13] where the code rate is ignored, the diversity gain in the coded performance is affected by the code rate $R$. In order to appropriately model the empirical results, a novel curve-fitting function is introduced as

$$
\mathcal{M}\left(R, \alpha^{2}\right)=\left(1+\sqrt[a]{\alpha^{2}}\right)^{b}
$$

where $a$ and $b$ are the parameters determined by $R$ in Table 3.1. For achieving the target bit error rate (BER), the minimum required SNR for MISO-SFN is defined [13] as

$$
\mathrm{SNR}_{\mathrm{MISO}}^{\mathrm{req}}=\mathrm{SNR}-10 \log _{10} \mathcal{M}\left(R, \alpha^{2}\right)[\mathrm{dB}]
$$

where $\mathrm{SNR}=1 / \sigma_{w}^{2}$ is the SNR for point-to-point SISO transmission. 


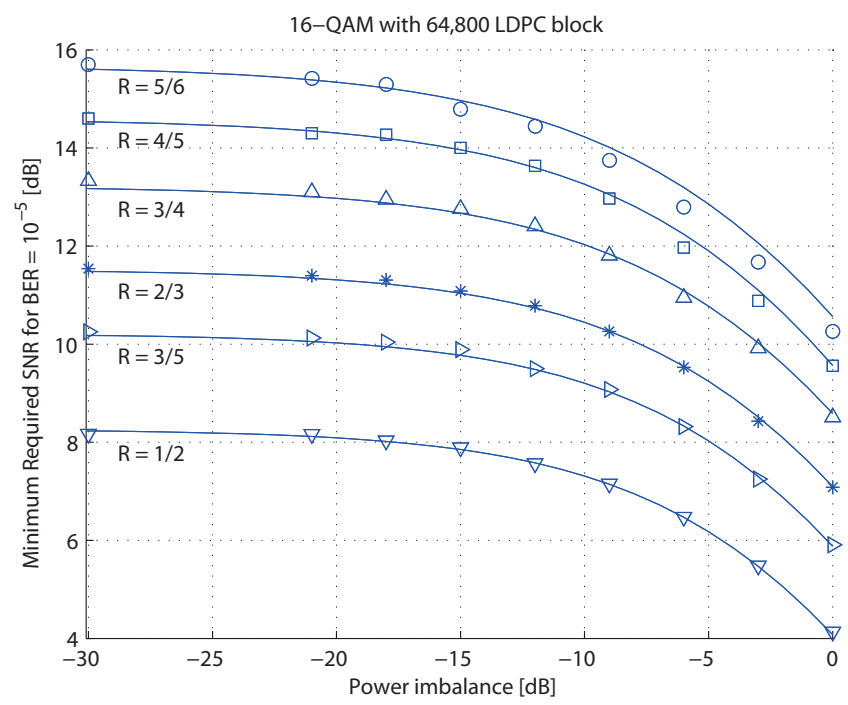

Fig. 3. Minimum required SNRs to achieve BER $=10^{-5}$ with respect to power imbalance in a 16-QAM system. 64-QAM with 64,800 LDPC block

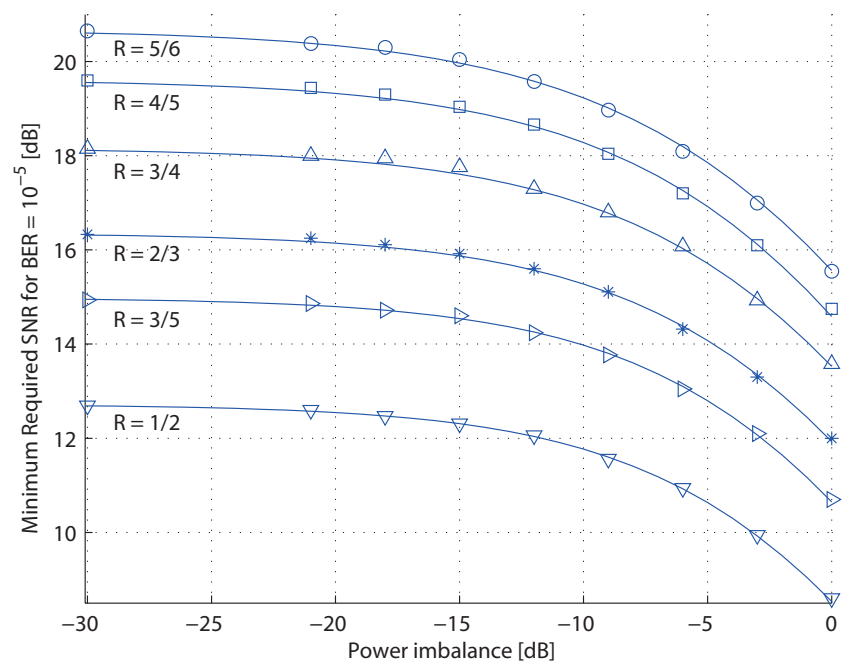

Fig. 4. Minimum required SNRs to achieve BER $=10^{-5}$ with respect to power imbalance in a 64-QAM system. 256-QAM with 64,800 LDPC block

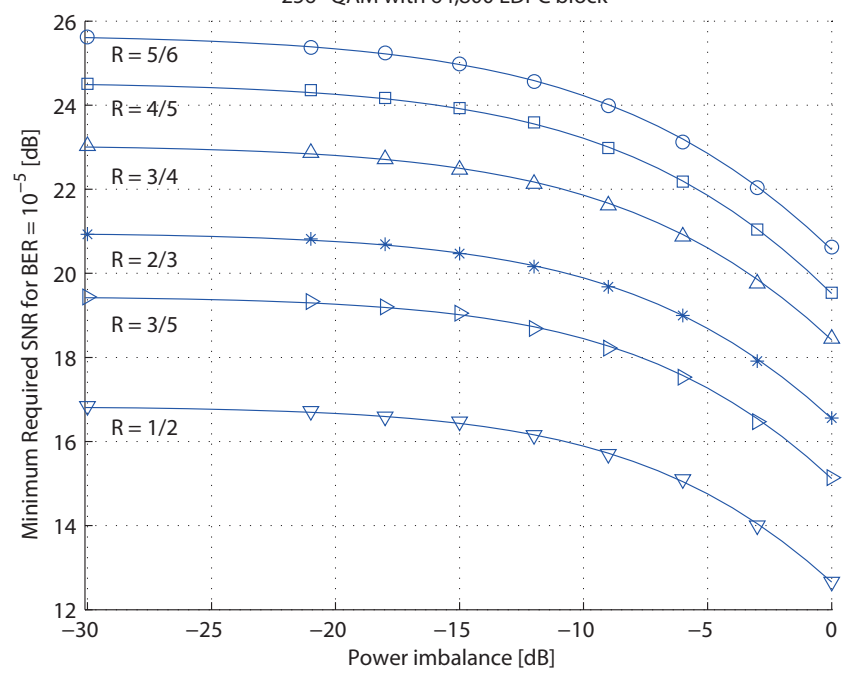

Fig. 5. Minimum required SNRs to achieve BER $=10^{-5}$ with respect to power imbalance in a 256-QAM system.

\begin{tabular}{c|c|c|c|c|c|c}
\hline$R$ & $\nabla 1 / 2$ & $\triangleright 3 / 5$ & $* 2 / 3$ & $\triangle 3 / 4$ & $\square 4 / 5$ & $\circ 5 / 6$ \\
\hline \hline$a$ & 1.30 & 1.32 & 1.36 & 1.41 & 1.44 & 1.51 \\
\hline$b$ & 1.39 & 1.44 & 1.47 & 1.54 & 1.67 & 1.70 \\
\hline
\end{tabular}

Tab. 1. Parameters $a$ and $b$ according to code rate for $\mathcal{M}$ used in DVB-T2 64,800 LDPC Block.

The simulation results of the minimum required SNRs for MISO-SFN are shown in Figs. 3 to 5 with respect to the received power imbalance. The corresponding curve-fitted lines in (14) are drawn in Figs 3 to 5, and they coincide very well with the simulation results. From the results, it is confirmed that the MISO-SFNG is higher for higher code rates and low power imbalance. This observation is in line with the results as conjectured in the ITU-R Report [4]. Interestingly, even though $\mathrm{SNR}_{\mathrm{MISO}}^{\text {req }}$ varies with modulation orders, the difference between $\mathrm{SNR}_{\mathrm{MISO}}^{\text {req }}$ and SNR, that is, the curve for MISO-SFNG is independent of the modulation orders.

\subsection{Loss in Frequency-Selective Fading Channel}

For the frequency-selective fading channel in the presence of the delay spread $H_{n, 2 k}^{1} \neq H_{n, 2 k+1}^{1}$ and $H_{n, 2 k}^{2} \neq H_{n, 2 k+1}^{2}$, and the determinant of the matrix $\mathbf{H} \mathbf{H}^{H}$ is not null. Consequently, the channel matrix $\mathbf{H}$ is no longer orthogonal and the matched filter receiver no longer provides the optimum solution.

From (10), the average power of the self-interference due to the delay spread can be expressed as

$$
\begin{aligned}
I_{1} & \\
= & \alpha^{2} E\left[\left|H_{n, 2 k+1}^{1 *} H_{n, 2 k+1}^{2}-H_{n, 2 k}^{1 *} H_{n, 2 k}^{2}\right|^{2}\left|X_{n, 2 k}\right|^{2}\right] \\
= & \alpha^{2} E\left[\left|H_{n, 2 k+1}^{1} H_{n, 2 k+1}^{2 *}-H_{n, 2 k}^{1} H_{n, 2 k}^{2 *}\right|^{2}\left|X_{n, 2 k+1}\right|^{2}\right] \\
= & \alpha^{2} E\left[\left|H_{n, 2 k+1}^{1}\right|^{2}\left|H_{n, 2 k+1}^{2}\right|^{2}\right]+\alpha^{2} E\left[\left|H_{n, 2 k}^{1}\right|^{2}\left|H_{n, 2 k}^{2}\right|^{2}\right] \\
& -\alpha^{2} E\left[H_{n, 2 k+1}^{1 *} H_{n, 2 k+1}^{2} H_{n, 2 k}^{1} H_{n, 2 k}^{2 *}\right] \\
& -\alpha^{2} E\left[H_{n, 2 k+1}^{1} H_{n, 2 k+1}^{2 *} H_{n, 2 k}^{1 *} H_{n, 2 k}^{2}\right] \\
\approx & \alpha^{2} E\left[\left|H_{n, 2 k+1}^{1}\right|^{2}\left|H_{n, 2 k+1}^{2}\right|^{2}\right]+\alpha^{2} E\left[\left|H_{n, 2 k}^{1}\right|^{2}\left|H_{n, 2 k}^{2}\right|^{2}\right] \\
& -\alpha^{2} E\left[H_{n, 2 k}^{1} H_{n, 2 k+1}^{1 *}\right] E\left[H_{n, 2 k}^{2 *} H_{n, 2 k+1}^{2}\right] \\
& -\alpha^{2} E\left[H_{n, 2 k}^{1 *} H_{n, 2 k+1}^{1}\right] E\left[H_{n, 2 k}^{2} H_{n, 2 k+1}^{2 *}\right] \\
= & 2 \alpha^{2}\left[1-\mathfrak{R e}\left(\rho_{1} \rho_{2}^{*}\right)\right]
\end{aligned}
$$

where the approximation in (17) is due to $|\eta| \ll$ $\min \left\{\left|\rho_{0}\right|,\left|\rho_{1}\right|\right\}$ from (7) which means that $H_{n, 2 k}^{1} H_{n, 2 k+1}^{1 *}$ and $H_{n, 2 k}^{2} H_{n, 2 k+1}^{2 *}$ are approximately independent. The correlation of the channel over two consecutive subcarriers can be written as $\rho_{1}=E\left[H_{n, 2 k}^{1} H_{n, 2 k+1}^{1 *}\right]$, and $\rho_{2}=E\left[H_{n, 2 k}^{2} H_{n, 2 k+1}^{2 *}\right]$. $\mathfrak{R e}(s)=\frac{1}{2}\left(s+s^{*}\right)$ takes the real part of complex number $s$.

We provisionally let $\rho_{1}=\rho_{2}=A_{c}(\Delta f)$ where $\Delta f$ is the frequency difference between two consecutive tones, and $A_{c}(\Delta f)$ is the Fourier transform of the power delay profile $A_{c}(\Delta \tau)$ where $\Delta \tau$ is the root mean squared (r.m.s.) delay spread representing the standard deviation of the power delay profile (PDP) [3]. These PDPs are considerably dif- 
ferent in appearance; interestingly, when $\Delta f \Delta \tau \ll 1$, all the idealized profiles lead to the same approximation of $1-\left|A_{c}(\Delta f)\right|^{2}=(2 \pi \Delta f \Delta \tau)^{2}$ [22]. Therefore, $I_{1}$ in (18) can be re-written as

$$
I_{1}=8 \pi^{2} \alpha^{2}(\Delta f \Delta \tau)^{2}
$$

\subsection{Loss due to Imperfect Channel Estimation}

From (11), the covariance matrix of the selfinterference due to imperfect channel estimation is given by $E\left[\mathbf{E}^{H} \mathbf{H X X} \mathbf{X}^{H} \mathbf{H}^{H} \mathbf{E}\right]=\sigma_{e}^{2}\left(1+\alpha^{2}\right) \mathbf{I}_{2}$. where $E\left[\mathbf{X X}^{H}\right]=\mathbf{I}_{2}$, because the average transmitted signal power is normalized. Hence, the average power of self-interference is

$$
I_{2}=\sigma_{e}^{2}\left(1+\alpha^{2}\right) \text {. }
$$

From (12), the covariance matrix of noise is given by $E\left[\left(\mathbf{H}^{H}+\mathbf{E}^{H}\right) \mathbf{W} \mathbf{W}^{H}(\mathbf{H}+\mathbf{E})\right]=\sigma_{w}^{2}\left(1+\alpha^{2}+2 \sigma_{e}^{2}\right) \mathbf{I}_{2}$. Thus, the average power of noise is

$$
W=\sigma_{w}^{2}\left(1+\alpha^{2}+2 \sigma_{e}^{2}\right) .
$$

\subsection{Net MISO-SFNG}

From (14), (16), (20) and (21), the average effective SINR for MISO-SFN is given by

$$
\operatorname{SINR}_{\mathrm{MISO}}=10 \log _{10} \frac{\mathcal{M}\left(R, \alpha^{2}\right)}{v I_{1}+I_{2}+W}[\mathrm{~dB}]
$$

where $v$ denotes the weighting factors, that are dependent on the code rate and modulation order and are used to adjust the effective interference compared to the measured data. From the property in (22), it is evident that any correlation between two antennas does not affect the average SINR. Finally, the net MISO-SFNG is defined by

$$
\mathrm{MISO}-\mathrm{SFNG}=\mathrm{SINR}_{\mathrm{MISO}}-\mathrm{SNR}[\mathrm{dB}] .
$$

\section{Verification and Discussion}

The validity of the derived formula in (23) is verified by comparing the measured results in [17][15]:

- 32K-FFT mode in a $8 \mathrm{MHz}$ bandwidth, i.e., the subcarrier spacing becomes $\Delta f=279 \mathrm{~Hz}$ [8],

- 64-QAM with FEC code rate 3/5, 2/3 and 3/4 are considered,

- the guard interval (GI) is chosen as $1 / 16$, i.e., $T_{g}=$ $224 \mu \mathrm{s}$ [8],

- $\sigma_{e}^{2}=10^{-3}$ is assumed according to the pilot pattern 2,

- SNR is set according to Table 2.14 in [4].
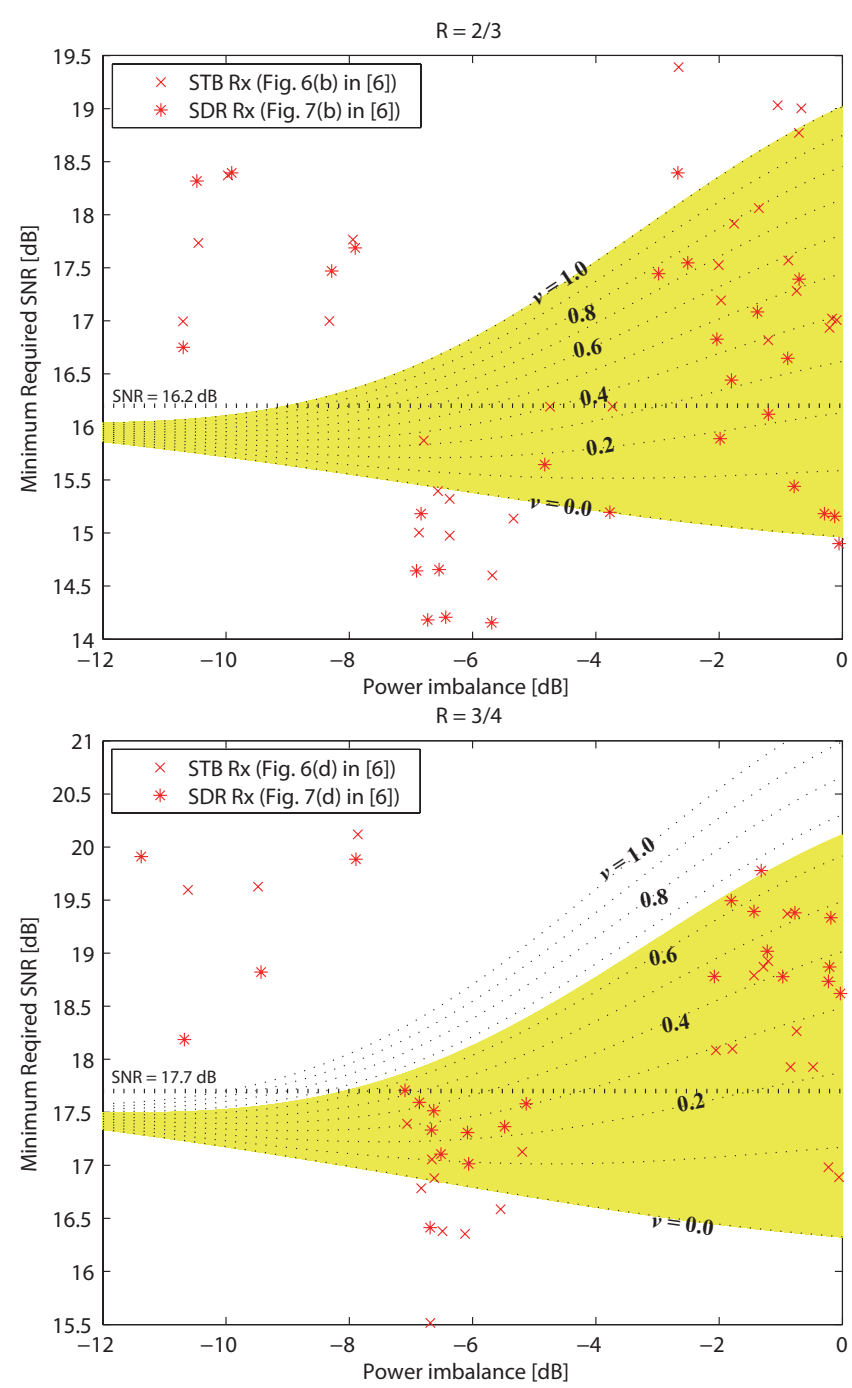

Fig. 6. Numerically obtained MISO-SFNG as a function of power imbalance and relative delay using (23) when (a) $R=2 / 3$. (b) $R=3 / 5$. $\mathrm{v}=1.0$. Minimum required SNR from the measured results reported in [17] and numerically obtained by the formula (solid line) as a function of the power imbalance. The SNR without an SFN is set to $17.7 \mathrm{~dB}$ for a 64-QAM code rate of 3/4 [4].

\subsection{Effect of the Weighting Factor $v$}

The minimum required SNRs obtained by field measurements in the southern part of Germany are plotted in Fig. 6 according to the power imbalance [17], comparing the measured results with the analytical results. The channel profile is not controllable and thus measured in various channel conditions; therefore, it can be observed that data from the field measurement are scattered. By adjusting the weighting factor $v$, e.g. $v=1.0$ for code rate $2 / 3$ in Fig. 6 (a) and $v=0.6$ for code rate $3 / 4$ in Fig. $6(b)$, the measured results coincide very well with the analytical results, where the influence of the delay effect is well reflected and the field measurement results can be analytically explained as follows. 


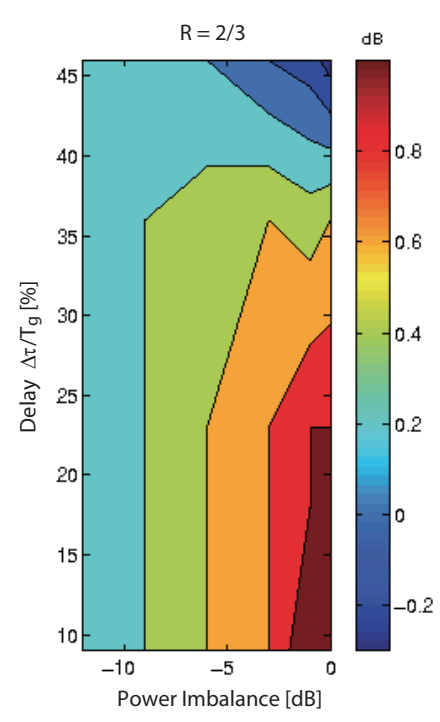

(a)

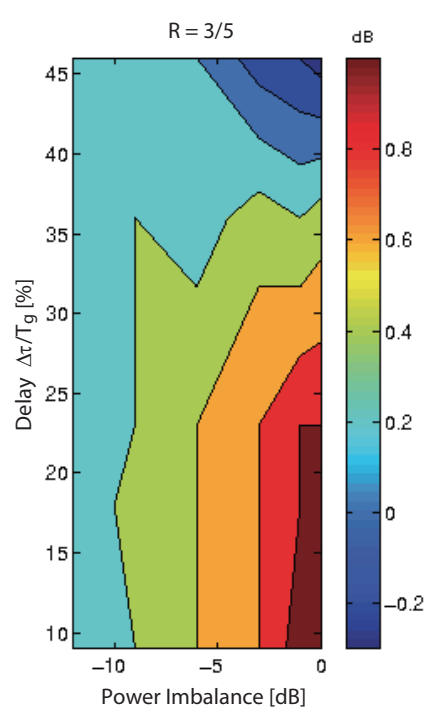

(b)
Fig. 7. Measured results as a function of power imbalance and relative delay in [15] when (a) $R=2 / 3$ (b) $R=3 / 5$.
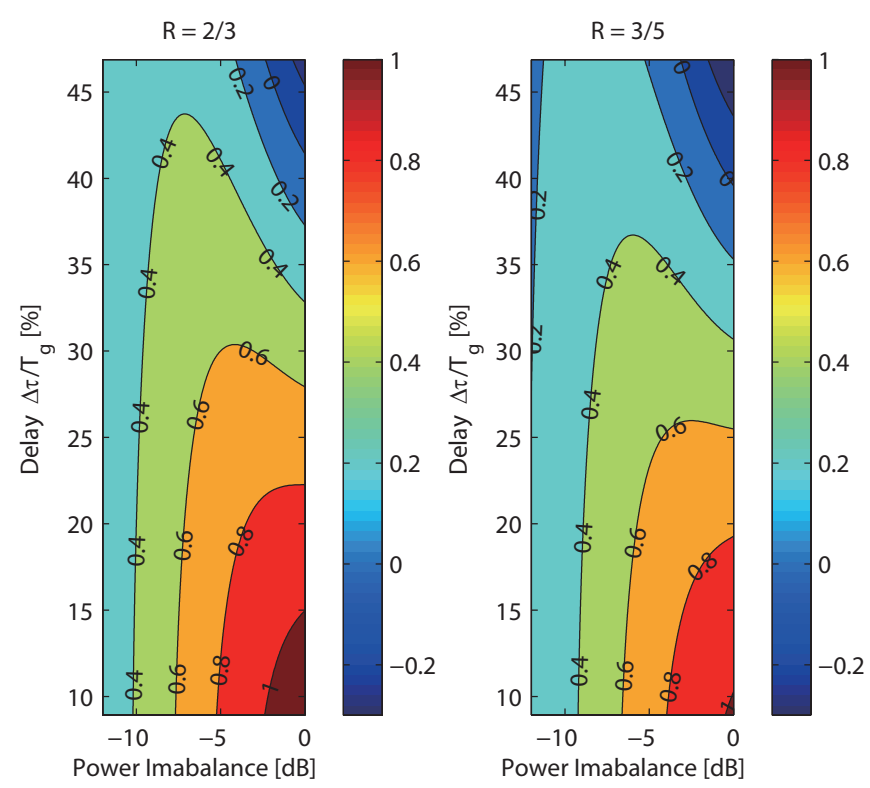

Fig. 8. Numerically obtained MISO-SFNG as a function of power imbalance and relative delay using (23) when (a) $R=2 / 3$ with $\mathrm{v}=0.90$ (b) $R=3 / 5$ with $\mathrm{v}=0.55$.

- In the case of $v=0.0$, no delay effect is considered so that the maximum net gain of MISO-SFN can be obtained. Thus, the line acts as the fixed lower bound.

- As the weight factor $v$ increases, the upper bound is increased so that the area between the lower and upper bound is expanded and the scattered data can be gradually included.

- If $v$ is fixed, the gap between the upper and lower bound increases as the power imbalance increases, and therefore, the negative gain of MISO-SFN is observed when the power imbalance is approximately above $-8 \mathrm{~dB}$.

\subsection{Verification of the Derived Formula}

For calculating the analytical results from (23), an identical MISO configuration in two-transmitter SFN has been considered in Tab. 2 [15].

By comparing Fig. 7 with Fig. 8, it is observed that the measured results coincide with the analytical results very well. In general, the MISO gain depends on the power imbalance, i.e., when the power imbalance is lower, the MISO gain is higher. As observed in Fig. 7, the analytical results in Fig. 8 satisfactorily reflect the influence of the relative delay between the received signals of different MISO groups.

In addition, by comparing Fig. 7(a) with Fig. 7(b), it can be confirmed that the effective MISO gain is also influenced by the code rate, i.e., the effective gain increases when the code rate is increased because the gain $\mathcal{M}(R, \alpha)$ is higher for high code rates.

The curve corresponding to the data obtained from the field trials and laboratory measurements is coarse, where the unmeasured data are obtained by interpolation. On the other hand, the curve generated by using the analytical formula in (23) is smooth and fine. Consequently, we can evaluate the MISO-SFNG and predict the MISO-SFN coverage in various configurations numerically.

\section{Conclusion}

In this study, we derived the formula for MISO-SFNG by considering the diversity gain as well as self-interferences caused by delay spread and imperfect channel estimation. The accuracy and effectiveness of the derived formula were verified by comparing the analytical results with the measured data. Consequently, the derived MISO-SFNG formula can be used to evaluate DVB-T2 network planning without laboratory or field measurements.

\section{Acknowledgments}

This work was supported by the ICT R\&D program of MSIP/IITP [2014-044-053-001, Development of the Advanced Broadcasting System based on FTN].

\section{References}

[1] MATTSSON, A. Single frequency networks in DTV. IEEE Transactions on Broadcasting, 2005, vol. 51, no. 4, p. 413-422. DOI: 10.1109/TBC.2010.2043897

[2] EBU BPN 066. Guide on SFN Frequency Planning and Network Implementation with Regard to T-DAB and DVB-T. European Broadcasting Union, 2005.

[3] GOLDSMITH, A. Wireless Communications. New York (USA): Cambridge University Press, 2005. DOI: http://dx.doi.org/10.1017/CBO9780511841224

[4] ITU-R Report BT. 2254. Frequency and Network Planning Aspects of DVB-T2. International Telecommunication Union, 2012. 
[5] PLETS, V., JOSEPH, W., ANGUEIRA, P., ARENAS, J. A. VERLOOK, L., MARTENS, L. On the methodology for calculating SFN gain in digital broadcast systems. IEEE Transactions on Broadcasting, 2010, vol. 56, no. 3, p. 331-339. DOI: 10.1109/TBC.2010.2051176

[6] ITU-R Recommendation BT.1306-6. Error Correction, Data Framing, Modulation and Emission Methods for Digital Terrestrial Television Broadcasting. International Telecommunication Union, 2012.

[7] ITU-R Recommendation BT.1877-1. Error Correction, Data Framing, Modulation and Emission Methods for Second Generation of Digital Terrestrial Television Broadcasting Systems. International Telecommunication Union, 2012.

[8] ETSI EN 302755 V1.3.1. Frame Structure, Channel Coding and Modulation for a Second Generation Digital Terrestrial Television Broadcasting System (DVB-T2), V1.3.1. European Telecommunications Standards Institute, 2012.

[9] JEON, S., BAEK, J.-S., KIM, J., SEO, J.-S. Analysis of the Network Gains of SISO and MISO Single Frequency Network Broadcast Systems. IEICE Transactions on Communications, 2014, vol. E97-B, no. 1, p. 182-189. DOI: http://dx.doi.org/10.1587/transcom.E97.B.182

[10] LANZA, M., GUTIERREZ, A. L., PEREZ, J. R., MORGADE, J., DOMINGO, M., VALLE, L., ANGUEIRA, P., BASTERRECHEA, J. Coverage optimization and power reduction in SFN using simulated annealing. IEEE Transactions on Broadcasting, 2014, vol. 60, no. 3, p. 474-485. http://dx.doi.org/10.1109/TBC.2014.2333131

[11] JEON, S., LEE, J., CHOI, J.-M., SEO, J.-S. Definition and properties of the erasure effect in single frequency network with two synchronized transmitters. IEEE Antenna and Wireless Propagation Letters, 2014, vol. 13, p. 439-442. DOI: 10.1109/LAWP.2014.2309699

[12] JEON, S., KIM, J., HAN, J.-S., SEO, J.-S. Formulating the net gain of an SISO-SFN in the presence of the erasure effect. IEEE Transactions on Broadcasting, 2015, vol. PP, no. 99. DOI: 10.1109/TBC.2015.2400812

[13] JEON, S., BAEK, J.-S., KIM, J., SEO, J.-S. Analysis on the network Gains of SISO and MISO single frequency network broadcast systems. IEICE Transactions on Communications, 2014, vol. E97-B, no. 1, p. 182-189. DOI: http://dx.doi.org/10.1587/transcom.E97.B.182

[14] TORMOS, M., TANOUGAST, C., DANDACHE, A., MASSE, D., KASSER, P. Modeling and performance evaluations of Alamouti technique in a single frequency network for DVB-T2. EURASIP Journal on Wireless Communications and Networking, 2013, vol. 78, no. 1. DOI: http://dx.doi.org/10.1186/1687-1499-2013-78

[15] MORGADE, J.ANGUEIRA, P. ARRINDA, A. ORDIALES, J. L. BRUGGER, R. FRANK, J. KUNERT, C. PFEFFER, R. DVB-T2 MISO/SISO receiver performance limits in single frequency networks: practical results. In The 8th IEEE International Symposium on Broadband Multimedia Systems and Broadcasting (BMSB). Uxbridge (UK), 2013. DOI: http://dx.doi.org/10.1109/BMSB.2013.6621718

[16] QI, J., ROBERT, J. CHEE, K. L. SLIMANI, M., ZOELLNER, J. DVB-T2 MISO field measurements and a calibrated coverage gain predictor. In The 7th IEEE international Symposium on Broadband Multimedia Systems and Broadcasting (BMSB). 2012. DOI: http://dx.doi.org/10.1109/BMSB.2012.6264296

[17] MORGADE, J., ANGUEIRA, P., ARRINDA, A., PFEFFER, R., STEINMANN, V., FRANK, J., BRUGGER, R. SFN-SISO and SFNMISO gain performance analysis for DVB-T2 network planning. IEEE Transactions on Broadcasting, 2014, vol. 60, no. 2, p. 272 286. DOI: http://dx.doi.org/10.1109/TBC.2013.2293852

[18] ETSI EN 302583 V1.1.1. Measurement Guidelines for DVB Systems. European Telecommunications Standards Institute, 2008

[19] Terrestrial tuner and demodulator. Nordig Unified Requirements for Integrated Receiver Decoders for Use in Cable, Satellite, Terrestrial and IP-Based Networks v2.4, Chapter 3.4. [Online] 2013. Available at: http://www.nordig.org/pdf/NorDig-Unified_ver_2.4.pdf.
[20] ETSI TS 102831 V1.2.1 Implementation Guidelines for a Second Generation Digital Terrestrial Television Broadcasting System (DVB-T2). European Telecommunications Standards Institute, 2012.

[21] ALAMOUTI, S. M. A simple transmit diversity technique for wireless communications. IEEE Journal on Selected Areas in Communications, 1998, vol. 16, no. 8, p. 1451. DOI: http://dx.doi.org/10.1109/49.730453

[22] CHUANG, J. C.-I. The effects of time delay spread on portable radio communications channels with digital modulation. IEEE Journal on Selected Areas in Communications, 1987, vol. 5, no. 5, p. 879. DOI: http://dx.doi.org/10.1109/JSAC.1987.1146591

[23] SALMAN, F., COSMAS, J., ZHANG, Y. Modelling and performance of a DVB-T2 channel estimator and equaliser for different pilot patterns. In 7th IEEE international Symposium on Broadband Multimedia Systems and Broadcasting (BMSB). 2012. DOI: http://dx.doi.org/10.1109/BMSB.2012.6264256

\section{About the Authors...}

Sungho JEON received the B.S. (with Honors) and M.S. degrees in Electrical and Electronics Engineering from Yonsei University, Seoul, Korea, in Aug. 2005 and Feb. 2007, respectively. Since March 2007, he has been with the Technical Research Institute at the Korean Broadcasting System (KBS), where he is currently a Research Engineer. Since March 2011, he has been pursuing a Ph.D. degree in electrical and electronics engineering with emphasis on $4 \mathrm{~K}$ UHDTV terrestrial digital broadcasting systems at Yonsei University.

Junghyun KIM received the B.S. and M.S. degrees in Electronics Engineering from Yonsei University, Seoul, Korea, in Feb. 2006 and Feb. 2008, respectively. Since March 2008, he has been with the Broadcast Technical Research Institute at Korean Broadcasting System (KBS), where he is currently a Research Engineer. His main research interests include the development of database for integrated RF measurement and analysis system.

Ha-Kyun MOK received his B.S. in Electrical Engineering from Seoul National University, Korea and M.S. in Electrical and Electronics Engineering from KAIST, Korea in 1980 and 1982 respectively. Since 1982, he has been working for KBS, Korea, for the development of Digital TV Broadcasting service. In 2013, he was a chief officer of TRI, KBS. His main research interests include production and transmission techniques for the broadcasting systems.

Jong-Soo SEO (corresponding author) received the B.S. degree in electronics engineering from Yonsei University, Seoul, Korea, in 1975, and the M.S. and Ph.D. degrees from the University of Ottawa, Ottawa, ON, Canada, in 1983 and 1988, respectively. He was with IDC and CAL, Canada, engaged in research on digital satellite communications and data broadcasting systems for six years. Since 1995, he has been with the Department of Electrical and Electronic Engineering, Yonsei University, where he is currently a Professor. His current research interests include mobile multimedia broadcasting and beyond fourth-generation mobile radio systems. 